\title{
Detection of aeolian transport in coastal images
}

\author{
Isaac A. Williams*, Kathelijne. M. Wijnberg, Suzanne. J.M.H. Hulscher \\ a Department of Water Engineering \& Management, Faculty of Engineering Technology, University of Twente, 7500AE Enschede, the Netherlands
}

\section{A R T I C L E I N F O}

\section{Keywords:}

Coastal processes

Streamers

Image processing

Remote sensing

Transport events

\begin{abstract}
A B S T R A C T
Optical remote sensing provides a low cost method of documenting surface conditions over extended periods of time. Its utility in resolving large scale aeolian transport events is examined in this paper. To this end, over 1800 images taken over a three month period by a stationary camera in a coastal setting are classified as containing/ not containing aeolian transport using both a manual and automated approach. In both cases transport is inferred by the identification of aeolian streamers within a given image. With regards to the manual approach, images were visually examined by three users. There was good agreement between the different users with $77.8 \%$ of transport identifications being unanimous. Comparison of the user results with measured transport highlights that observations of transport in images coincide with increased particle counts. The particle counts indicate that transport is dominated by a series of discernible transport events. Transport is observed in images throughout the duration of these events. Manually processing the images is a laborious process. A method is presented which allows for the automated detection of images containing transport. The method utilises 2DDFTs to exploit the linear appearance of streamers in images. Relative to the manual classifications which serve as ground truth, the method performs well in identifying images containing transport with values of precision, recall and $\mathrm{F} 1$ score $\geqslant 0.85$.
\end{abstract}

\section{Introduction}

Dunes play an important role in protecting low lying coastal areas against coastal flooding. Their growth and recovery after storm erosion requires the supply of sediment from the subaerial beach by winddriven processes. Whilst recent approaches in coastal management try to utilise these processes to promote dune growth (Stive et al., 2013), our ability to predict the sediment supply over relevant timescales (i.e. monthly to annual) remains poor.

Across these timescales, sediment supply is typically calculated by applying variants of the traditional, semi-empirical process-based transport equations (e.g. Bagnold, 1941; Kawamura, 1951) to routine meteorological data (e.g. Hsu, 1974). At process-scale, these equations, which assume steady-state transport, over predict transport rates in coastal settings (Sherman and Li, 2012). In part, this is due to their failure to (appropriately) account for a range of conditions which characterise coastal environments, e.g. moisture (e.g. Wiggs et al., 2004; Davidson-Arnott and Bauer, 2009) and lag deposits (e.g. van der Wal, 1998; Hoonhout and de Vries, 2017b). In conjunction with the spatial constraints placed by beach width (e.g. Delgado-Fernandez, 2010), these factors inhibit the evolution of the transport system to fully steady state conditions (Davidson-Arnott and Bauer, 2009). Unsurprisingly, when these transport equations are applied over longer time scales they overestimate sediment supply (e.g. Keijsers et al., 2014). Consequently, the calculated sediment supply is often regarded as an upper bound and is termed potential transport. When considered over monthly timescales, the potential transport can exhibit conflicting trends with the observed supply (e.g. Davidson-Arnott and Law, 1996). In part, this a consequence of a significant proportion of the calculated potential transport being contributed by a limited number of significant winds events, not all of which contribute to the actual observed sediment supply. Though a few major transport events are likely to be responsible for the majority of sediment supply to the dunes, the strongest winds events do not always result in the significant transport due to the cooccurrence of conditions such as precipitation events, storm surges etc., which shut the transport system down (Delgado-Fernandez and Davidson-Arnott, 2010; Delgado-Fernandez, 2011). The interaction of the various supply limiting conditions make it difficult to predict when a wind event will result in a significant transport event.

A lack of understanding as to when the transport system is shut down hinders our ability to predict longer term sediment supply. Consequently, long-term datasets that are able to document the occurrence of large scale transport events are desirable. The linkage of such datasets to surface, tidal, and atmospheric conditions, can provide

\footnotetext{
* Corresponding author.

E-mail address: i.a.williams@utwente.nl (I.A. Williams).
} 
insight into conditions that are (un-) favourable for wind-driven transport. The creation of datasets with sufficient spatial and temporal coverage using traditional in situ techniques is challenging. In this regard, coastal imagery, which documents surface conditions over a wide area during daylight hours for however long the camera system is operational, has a number of advantages (e.g. Holman and Stanley, 2007). The quantitative use of images requires that relevant physical processes leave an optical signal that can be exploited. Whilst progress has been made in using images to quantify factors that may influence the occurrence of transport events, e.g. surface moisture (Darke et al., 2009; Delgado-Fernandez et al., 2009) and shoreline position, (e.g. Aarninkhof et al., 2003; Hoonhout et al., 2015) and hence fetch length, their application in identifying transport events remains limited.

One of the ubiquitous features of large scale transport events in coastal environments is the presence of regions of intense saltation activity and high sediment concentrations, known as streamers. With widths in the order of $0.2 \mathrm{~m}$, length scales in excess of $50 \mathrm{~m}$, and horizontal separations of $1 \mathrm{~m}$, streamers snake across the surface propagating in the downwind direction (Baas and Sherman, 2005), contributing significantly to the total transport rates (Ellis et al., 2012). As highlighted in Fig. 1a, streamers are frequently observed within coastal images, particularly in areas where the underlying sediment is relatively moist. This provides relatively strong contrast differences with the dry and thus lighter sediment of the streamers. The identification of streamers in instantaneous snap-shot images can therefore confirm the occurrence of wind-driven transport at a given moment in time. In contrast, the rapid movement of streamers means they are averaged out of time averaged images. This is illustrated in Fig. 1b. The application of images in the study of streamers remains limited. Exploratory work, indicates that they may have utility in resolving the spatial and temporal structure of streamers (Sherman et al., 2013). The manual identification of streamers in images has been used to document the occurrence of transport events (van and Wijnberg, 2016). Manual identification is a slow process, and it remains unclear how user bias influences the identification of streamers.

The aim of this paper is to examine the utility of routine coastal imagery in resolving large scale wind-driven transport events. To this end, a number of users manually classified $>1800$ coastal images as containing/ not containing wind-driven transport. The subjectivity involved in manual classification is examined and the classified images are related to measured transport. A method is presented which seeks to classify images containing transport in an automated manner.

The remainder of this paper is organised as follows: The field site and available data are introduced in Section 2. Section 3 details the steps adopted to classify images manually and in an automated manner. The results of both approaches are given in Section 4 and discussed in Section 5. Finally, conclusions are drawn in Section 6.

\section{Field site $\&$ data}

Coastal images from the Sand Motor are considered in this study. The site is an artificial peninsula located on the west coast of the Netherlands. Upon its completion in 2011, it consisted of $21 \mathrm{Mm}^{3}$ of mined offshore sediment distributed over alongshore and cross-shore distances of $2.4 \mathrm{~km}$ and $1 \mathrm{~km}$ respectively (Stive et al., 2013). The project aims to utilise natural processes to redistribute this sediment, providing coastal protection along the adjacent coastline over decadal timescales. The supply of sediment to dunes by wind-driven processes is important to this. The topography of the site in October 2014 is shown in Fig. 2.

A camera station is located at the centre of the site and has been collecting images since 2013. The station is part of the larger network of Argus stations which collect coastal image data in standardised way (Holman and Stanley, 2007). Argus stations are primarily designed for use in nearshore studies and are typically mounted $\approx 40 \mathrm{~m}$ above the beach surface to provide optimal coverage for these purposes. The east facing camera overlooking the lagoon area is considered in this study (see Fig. 2). The lagoon area is infilling as a consequence of wind-driven transport (Hoonhout and de Vries, 2017a). When fully operational, the camera collects timestamped RGB snapshot (snap) and time exposure (timex) images concurrently every 30 min during daylight hours. The latter image type represents the mathematical mean of all images taken over a 10 min window.

A Campbell Scientific meteorological station is mounted on top of the camera tower (height $\approx 44 \mathrm{~m}$ ), providing measurements of minutely averaged wind speeds and directions. Tidal levels are measured at a tide gauge located $10 \mathrm{~km} \mathrm{NW}$ of the site every $10 \mathrm{~min}$. The site is subject to LiDAR surveys every 6 months, providing regular topographic measurements of the subaerial beach and dunes at $2 \times 2 \mathrm{~m}$ resolution. In situ aeolian transport measurements are available between September 17 and October 23, 2014. During this period Hoonhout and de Vries (2017b) deployed a series of masts equipped with Wenglor forks, providing particle count measurements at heights of $0.03 \mathrm{~m}, 0.1 \mathrm{~m}$ and $0.25 \mathrm{~m}$ above the surface, measured at a frequency of $1 \mathrm{~Hz}$. The spatial arrangement of masts varied through time. The mast closest to the field of view for each arrangement is shown in Fig. 2.

Given the available transport data, images taken during daylight hours in August, September and October 2014 are considered in this
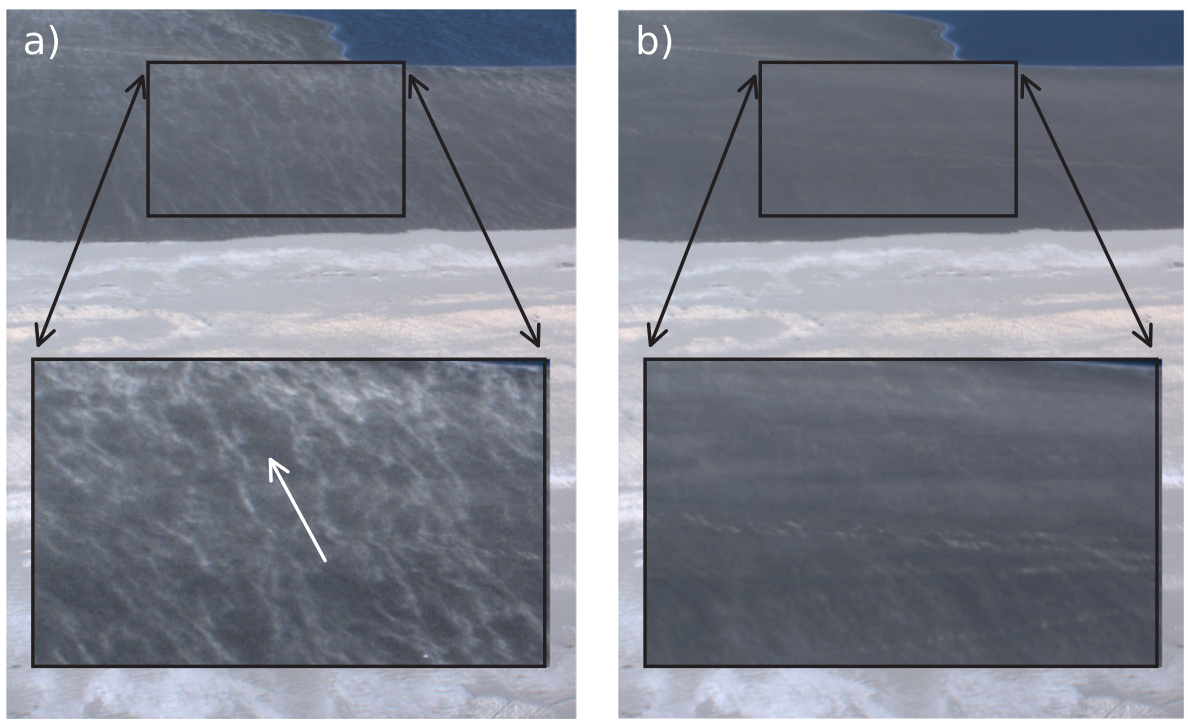

Fig. 1. (a) Streamers in a snap shot image at the Sand Motor. They are most noticeable in the upper half of the image (intertidal area) where there is a good contrast with underlying moist sediment. This area is enlarged in lower half of the image. Streamers are of a lighter colour than the underlying beach. The white arrow highlights their propagation direction. (b) Concurrent time exposure image (average of all images taken within $10 \mathrm{~min}$ window) in which the streamers are averaged out. 


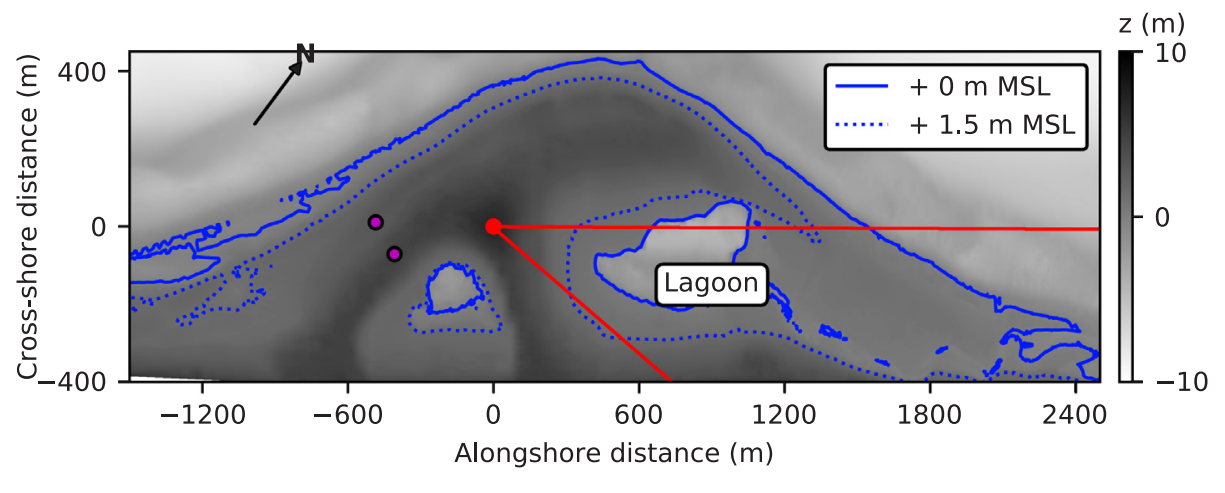

Fig. 2. Sand Motor in October 2014. Blue lines illustrate $+0 \mathrm{~m}$ and $+1.5 \mathrm{~m}$ MSL contours. The latter approximates high tide level. Red dot denotes location of the camera and meteorological stations. Solid red lines bound field of view of the considered camera. Colours correspond to topography/ bathymetry of site relative to MSL. Magenta dots denote locations of the considered Wenglor deployments of Hoonhout and de Vries (2017b). (For interpretation of the references to colour in this figure legend, the reader is referred to the web version of this article.)

study. As the camera faces east, images taken in the early morning were often characterised by severe lens flare. These images are removed from the analysis. In total 1811 concurrent pairs of snap and timex images are examined.

\section{Methodology}

\subsection{Manual classification}

Three users manually examined all images and classified them as containing or not containing transport (i.e. binary classification). Transport was inferred from the observation of streamers within the snap images. A graphical user interface was created to aid this process and minimise user error. The interface allowed the user to select one or more timestamps corresponding to the concurrent snap and timex image pairs. For each timestamp, the interface displayed a loop of: (1) the timex image relating to the previous timestamp (2) the timex image relating to the selected timestamp and (3) the snap image relating to selected timestamp. The images were labelled so it was clear which image was being observed. Through consideration of these three images the user made an informed decision as to whether they observed transport. No information relating to wind-speed was provided. Consequently, checks were entirely visual. The user checked a box if they observed transport which updated an underlying file. The user was able to process as many timestamps as they wanted in a single session and was free to choose a selection of non-consecutive timestamps if desired. However, the routine always processed the timestamps chronologically. The user could stop processing the images when desired. It took around $24 \mathrm{~h}$ for each user to process the dataset.

In order to relate image transport observations to in situ measured transport, the measurements of Hoonhout and de Vries (2017b) from the mast closest to the field of view of the camera are considered. Particle counts are summed over the three elevations, averaged over the preceding $10 \mathrm{~min}$ and rounded to the nearest integer value.

\subsection{Automated classification}

Using standard photogrammetric relationships, $u v$ image coordinates can be mapped to real world $x y z$ positions (e.g. Hartley and Zisserman, 2004). This allows for the creation of plan view images of a beach defined in real world spatial scales. When streamers pass through these images they often appear as linear image features. Detecting linear image features represents a texture classification problem, which can be examined through the application of 2D Discrete Fourier Transforms (2D-DFT's) (Josso et al., 2005). An image containing a regular periodic signal will result in two distinct peaks in the corresponding Fourier amplitude spectra, located in opposing quadrants. This is illustrated in Fig. 3, which presents a cosine wave (a) and its corresponding amplitude spectra (b). The dashed line which passes perpendicular to line connecting the two peaks can be used to estimate the orientation of the crests of the waveform about the $y$ axis, i.e. the a)

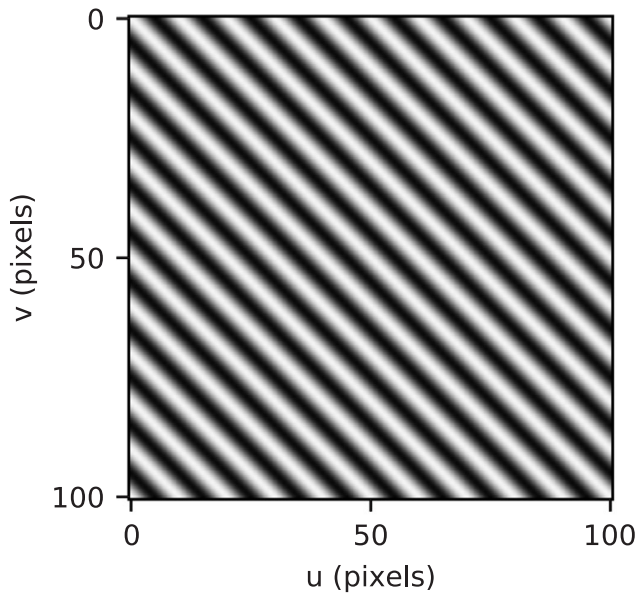

b)

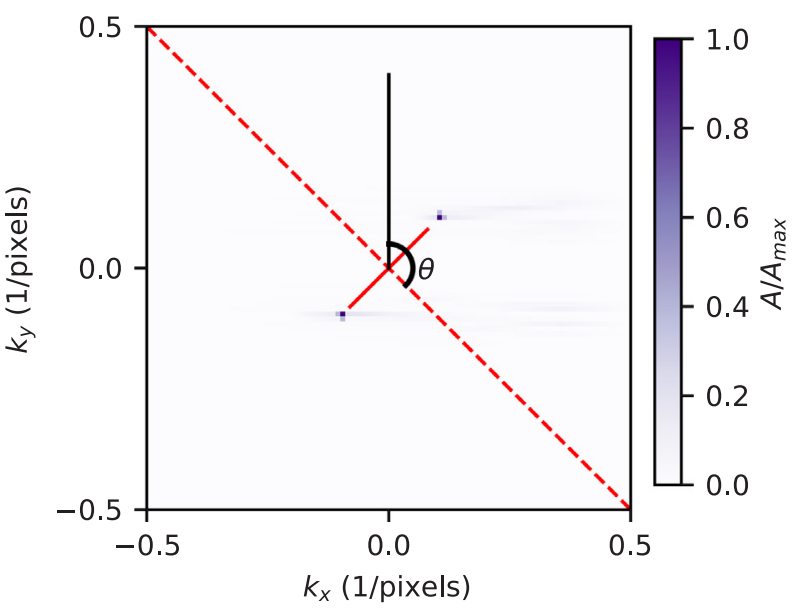

Fig. 3. (a) Input wave signal (b) corresponding amplitude spectra normalised by peak amplitude. Amplitude spectra is characterised by two peaks located in opposing quadrants. Solid red line connects peaks. Dashed line perpendicular to the solid red line. $\theta$ gives orientation of solid red line about $y$ axis. (For interpretation of the references to colour in this figure legend, the reader is referred to the web version of this article.)

angle $\theta$. Streamers can be considered as the crests of the wave shown in Fig. 3a. Hence, their propagation direction is given by either $\theta$ or $\theta+\pi$. As streamers snake across the surface, the resulting amplitude spectra will be distributed about the dashed line in Fig. 3b.

Section 3.2.1 outlines a simple Fourier based approach to identify the presence of streamers in plan view images. The method utilises concurrent $50 \times 50 \mathrm{~m}$ snap and timex RGB plan view images of frame size $N_{u}, N_{v}=[256,256]$, in conjunction with the measured wind direction. Section 3.2.2 extends of this approach to coastal images to determine if wind-driven transport is observable. 


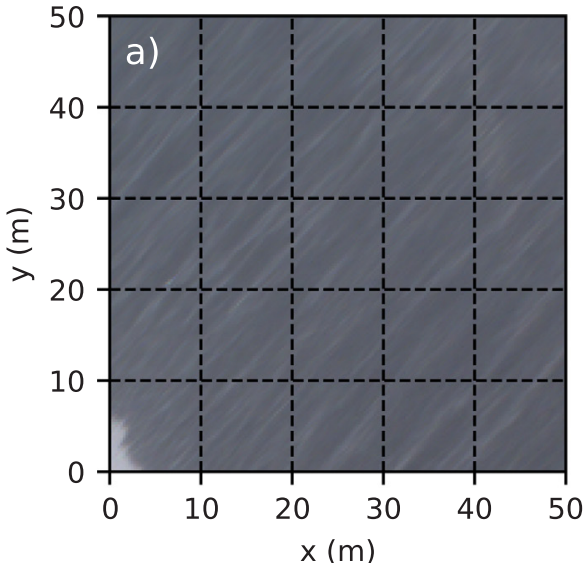

$\Delta$

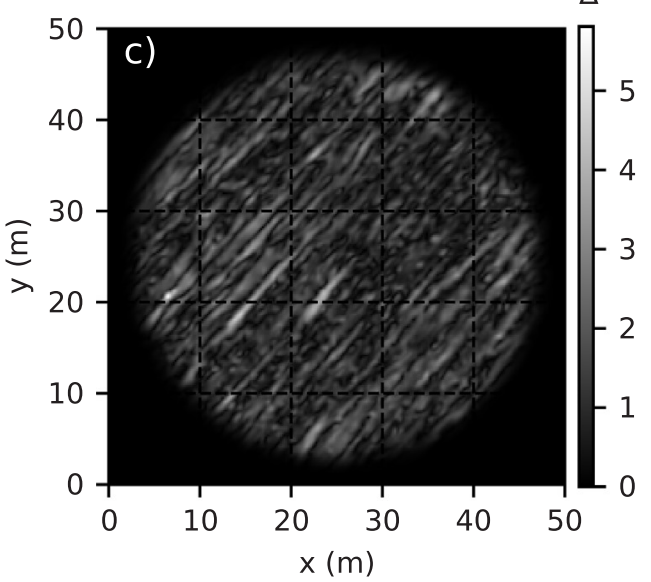

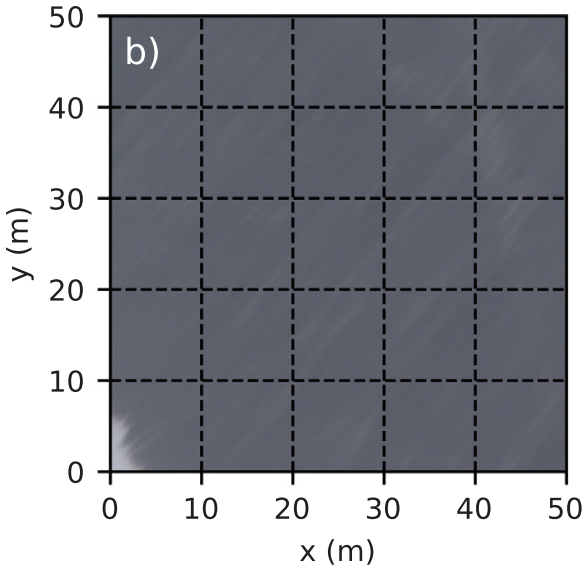

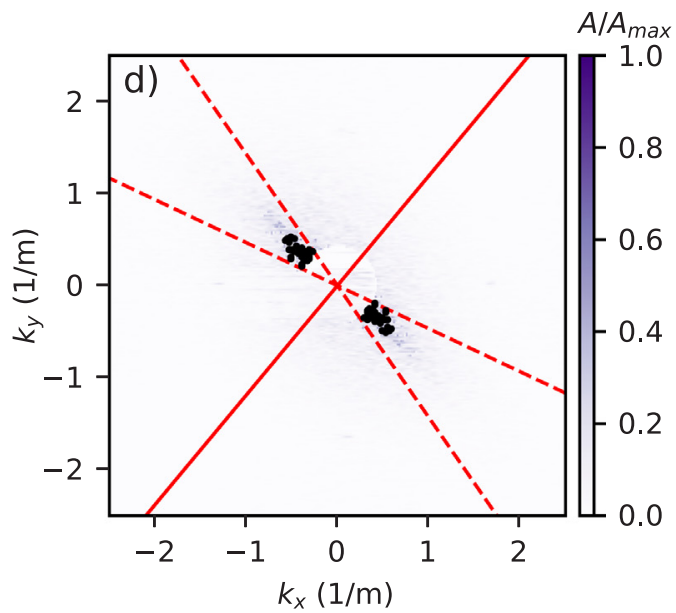

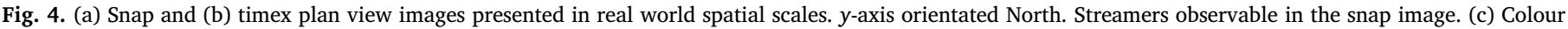

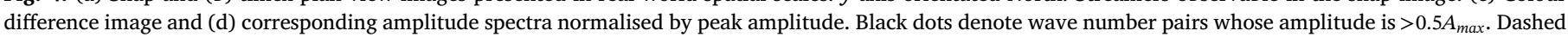
lines bound wavenumber pairs that lie $\pm 15^{\circ}$ from the best fit regression. Solid line perpendicular to regression line gives orientation of streamers.

\subsubsection{Identifying streamers in a plan view image}

Fig. $4 \mathrm{a}$ and $\mathrm{b}$ present concurrent snap and timex plan view images extracted from an image taken by the camera considered in this study. Streamers are observable within the snap plan view image. In order to identify their presence in automated manner, the plan view images are first transformed from RGB to the more perceptually uniform CIE $\mathrm{L}^{*} \mathrm{a} * \mathrm{~b} *$ colourspace. In this colourspace the $\mathrm{a*}$ and $\mathrm{b} *$ channels denote $\mathrm{a}$ pixels colour and the $L^{*}$ channel describes its lightness. Each channel is then tapered to its mean value using a 2D tapered cosine window and high pass filtered to remove potential large scale illumination variations. To account for lighting differences between the two images the mean of each L*a*b* channel is subtracted from the timex image and replaced by the corresponding measure from the snap image.

A colour difference image, $\Delta$, is calculated from the two plan view images. This represents the euclidean distance between pixel values and is defined according to:

$\Delta=\sqrt{\left(\mathrm{L}_{s}^{*}-\mathrm{L}_{t}^{*}\right)^{2}+\left(\mathrm{a}_{s}^{*}-\mathrm{a}_{t}^{*}\right)^{2}+\left(\mathrm{b}_{s}^{*}-\mathrm{b}_{t}^{*}\right)^{2}}$

where the subscripts $s$ and $t$ stand for snap and timex respectively. The resulting colour difference image is shown in Fig. 4c. The colour difference image filters out static image information and limits the identification of persistent linear image features unrelated to streamers e.g. bedforms.

A 2D-DFT is then applied to the colour difference image. Fig. 4d shows the amplitude spectra corresponding to the colour difference image given in Fig. 4c. The presence of the streamers forces the spectra to have a preferred orientation. To determine the orientation, wave number pairs whose amplitudes are $>0.5 A_{\max }$, where $A_{\max }$ is the peak amplitude of the spectra, are selected. These are highlighted as black circles in Fig. 4d. A linear regression is conducted through these points which has the form $k_{y}=m \cdot k_{x}$, where $m$ is the gradient. The line perpendicular to this is used to calculate the direction of propagation of streamers. The gradient of this line is given by $m_{\text {dir }}=-1 / m$. This is presented as the solid line in Fig. 4d. Two possible streamer orientations are obtained from $m_{d i r}, \theta_{d i r, \min }$ and $\theta_{d i r, \text { max }}$, as it is not possible to determine they are orientated e.g. the NW or the SE. The two directions are given by:

$\theta_{d i r, \min }=\left\{\begin{array}{cc}\frac{\pi}{2}-\arctan \left(m_{d i r}\right) & \text { if } m_{d i r}>0 \\ \frac{\pi}{2}+\left|\arctan \left(m_{d i r}\right)\right| & \text { if } m_{d i r}<0\end{array}\right.$

$\theta_{d i r, \text { max }}=\pi+\theta_{d i r, \min }$

The most appropriate value is taken to be the one closest to measured wind direction.

The regression method will begin to fail as the streamer direction approaches $0 / 90 / 180 / 270^{\circ}$ as, from the regressions perspective, the selected wavenumber pairs become independent of each other. To circumvent this, the colour difference metric is rotated by $45^{\circ}$, if the measured wind direction is $\pm 15^{\circ}$ of these angles. The values of $m_{d i r}$ are then rotated back to it's original orientation, and the appropriate value of $\theta$ determined.

The above method always yields a direction, however its reliability depends on the quality of the regression. Consequently, two checks are implemented. The first requires the regression to have $r^{2} \geqslant 0.75$. The 
second criteria is based on "energy" considerations. To this end, wave number pairs lying within $\pm 15^{\circ}$ of the regression line are selected. This is illustrated by the dashed lines in Fig. 4f. Amplitudes corresponding to these wavenumber pairs are then squared, summed and divided through by sum of all squared amplitudes. If there is no directional signal, amplitudes are expected be largest at the centre of the spectra and decrease radially towards the edges. In such a case, a value of $(2 \cdot 30) / 360 \approx 0.16$ is anticipated. Consequently, a value of $>0.3$ is interpreted as indicating a preferred orientation. If both these checks are passed, the image is taken as containing a linear image feature, and hence streamers. The determined orientation represents the average direction of streamers over the $50 \times 50 \mathrm{~m}$ area considered.

\subsubsection{Application to image dataset}

In order to classify wind-driven transport in a concurrent snap and timex image pair from the image dataset as containing/ not containing transport, a series of plan view images of the subaerial beach are extracted to which the method outlined above is applied. The location the extracted plan view images within the image frame are based on real world spatial considerations. To this end, the camera pose is solved and a 2D grid is created in the local coordinate system of the camera. Grid points are introduced at a number of fixed radial distances from the camera origin with the spacing between radial lines set to $20 \mathrm{~m}$. Along a given radial line a series of points are added such that the arc length between consecutive points is also equal to $20 \mathrm{~m}$. The grid is then rotated to real world $x y$ coordinates. Bounding boxes which correspond to real world $x y$ areas of $50 \times 50 \mathrm{~m}$ orientated about true north are defined about each point. The $z$ values of the grid points and their corresponding bounding box are determined from the nearest $x y$ neighbour from the closest topographic survey in time. The $x y z$ coordinates are then transformed to image coordinates. Only grid points whose bounding box lies within the image frame are considered further.

For a given bounding box, a perspective transformation is applied to both the snap and timex images to create plan view images of dimensions $N_{u}, N_{v}=[256,256]$. The NE/NW/SW/SE corners of the bounding box are located in top left/top right/bottom right/bottom left corners of the new image frames. To ensure that a bounding box has sufficient spatial resolution to resolve streamers four distances are considered; $\Delta u, \Delta v, l_{1}$ and $l_{2} . \Delta v /(\Delta u)$ corresponds to the pixel distance between the maximum and minimum value of the vertical (/ horizontal) image coordinates of the outer bounding box corners. $l_{1}$ and $l_{2}$ are the euclidean pixel distances between the NE- SW corners and NW-SE corners (see Fig. 5a). For the considered camera, $\Delta v$ is always less than $\Delta u$, and reduces at quicker rate with increasing radial distance. The ensuing reduction in the ratio $l_{2} / l_{1}$ leads to increased uncertainty in the orientation of streamers as observed in the plan view images. Consequently, subaerial plan view images are extracted if (1) $\Delta v \geqslant 128$ or (2) $\Delta v \geqslant 100$ and $l_{2} / l_{1}>0.15$. Fig. 5 shows an example of the grid created for the camera considered in the current study showing the distribution of points in (a) image and (b) real world space. Only grid points which pass the above tests are shown. The grid points extend across $x y$ radial distances of $140-380 \mathrm{~m}$.

The method outlined in Section 3.2.1 uses linear image features to infer the presence of streamers. Consequently, care is taken to avoid the identification of linear image features that are unrelated to streamers. For example, people, who are typically only observable in snap images, become warped across the plan view images and can force directionality in the resulting amplitude spectra. Consequently, plan view images are discarded from the analysis if there are strong colour differences between the snap and timex images (maximum $\Delta$ value $>20$ ). Further, the relatively linear shadow cast by the camera tower can force directionality in the amplitude spectra of portions of images through which it passes. As the images are timestamped, the position of the shadow within a given image frame is estimated (Bretagnon and Francou, 1988) and the effected plan view images removed.

The method outlined above is applied to all images in the dataset. A snap and timex image pair is always marked as containing transport if linear image features are identified in at least two of the extracted plan view images. An image pair containing one plan view image with linear image features is marked as containing transport, only if there is an image $\pm 1 \mathrm{~h}$ of the given timestamp where linear image features identified in at least two plan view images. In order to evaluate the performance of the method, the results are compared to the user results which serve as ground truth.

\section{User results}

\subsection{Cross user results}

The cross user counts of images containing transport across the entire image dataset are summarised in Table 1 . The majority user results require at least two users to observe transport in a given image. The users observed transport in images over comparable wind speed ranges. For example, the minimum wind speed at which a user observes transport, $u_{\text {thresh }}$, is largest for User 1 . However, User's 2 and 3 only observed transport in 4 and 6 images with concurrent wind speeds

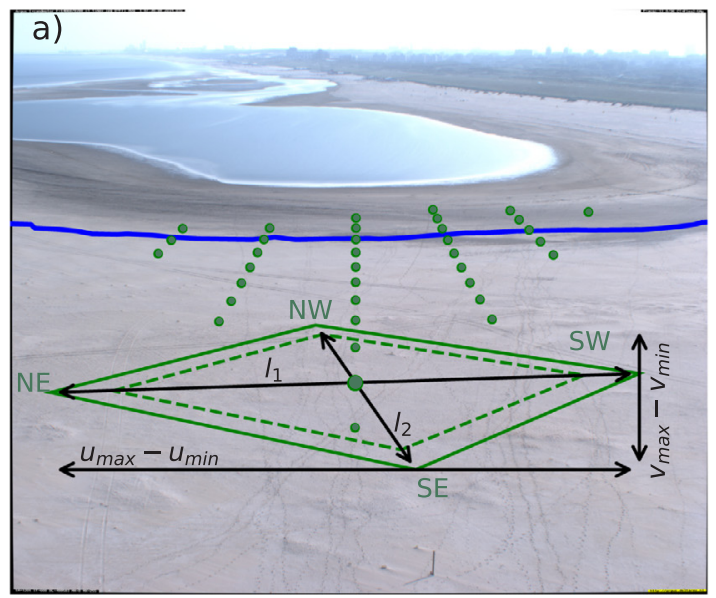

b)

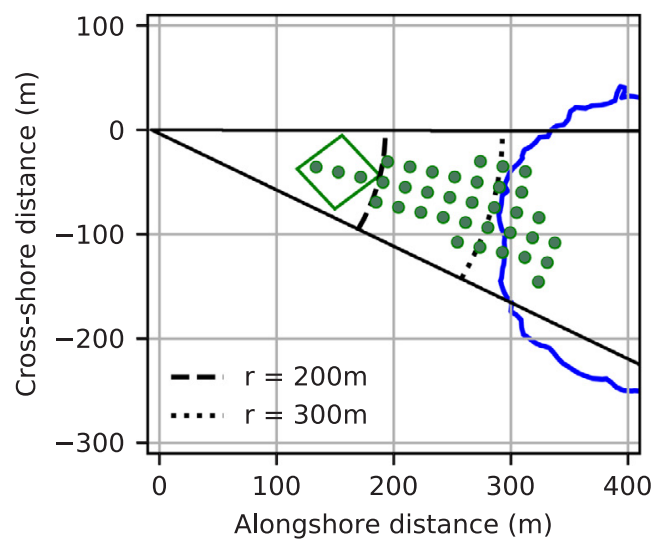

Fig. 5. (a) Illustration of grid within an image. Dots denote grid points about which plan view images are extracted. Green polygon deNotes $50 \times 50 \mathrm{~m}$ area surrounding a grid point which is used to construct the plan view image. The compass direction of the corners of the polygon are given in green text. Black arrows denote the considered dimensions in determining whether plan view image is processed. (b) The same grid in real world space. Solid black line bounds field of view of camera. Dashed/ dotted lines denote fixed radial distances from the camera origin about which grid points are introduced. Blue lines correspond to $+1.5 \mathrm{~m}$ MSL contour in both. 
Table 1

Summary of user results. $u_{\text {thresh }}$ corresponds to the minimum wind speed at which transport is identified. Majority decision requires at least two users to identify transport for a given image frame.

\begin{tabular}{llll} 
& \multicolumn{2}{c}{ Counts } & $u_{\text {thresh }}(\mathrm{m} / \mathrm{s})$ \\
\cline { 2 - 3 } & Transport & No transport & \\
\hline User 1 & 244 & 1567 & 9.91 \\
User 2 & 265 & 1546 & 4.34 \\
User 3 & 295 & 1516 & 7.67 \\
\hline Majority & 267 & 1544 & 8.56 \\
\hline
\end{tabular}

lower than this threshold.

Overall, User's 1, 2 and 3 observed transport in 13.5\%, 14.6\%, and $16.3 \%$ of all images. With regards to transport observations, unanimous, non-unanimous and potential transport counts are considered. Unanimous counts correspond to images in which all users observe transport, whilst non-unanimous counts relate to images where only one/ two user(s) observe transport. Potential counts correspond to images in which at least one user observed transport (i.e. can be either a unanimous or non-unanimous count). There are a total of 302 potential counts, of which $235(77.8 \%)$ correspond to unanimous counts, 32 $(10.6 \%)$ to non- unanimous counts where two users observed transport and $35(11.6 \%)$ to non-unanimous counts where only one user observed transport. The unanimity of transport identifications appears to be dependent on wind speed. This is illustrated in Fig. 6, which presents a histograms of the potential and unanimous counts, binned according to wind speed. The ratio of the two counts indicates that there is better agreement between users as wind speed increases.

\subsection{Comparison with measured transport}

In this section the majority user results are related to the transport measurements of Hoonhout and de Vries (2017b). In total, 499 images coincided with the field campaign, with transport being observed in 88 images.

Fig. 7a presents a scatter plot of wind speed and particle counts measured concurrently with the considered images. During mild wind conditions transport is limited, with no nonzero particle counts measured when the wind speed is less than the majority user threshold. In total 59 images coincide with nonzero particle counts, of which 44 (74.6\%) are associated with observable transport. Consequently, nonzero particle counts are typically associated with observable transport. Further, nonzero particle counts associated with observable transport are typically in excess of those corresponding to non-observable

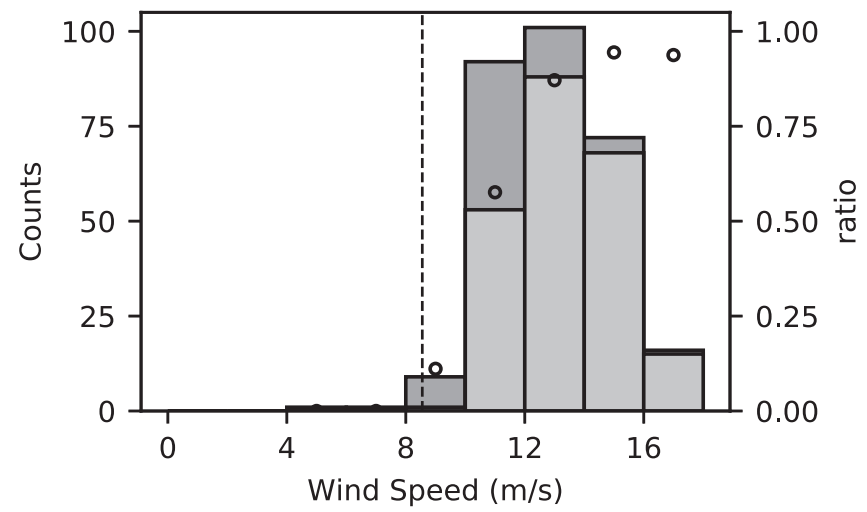

Fig. 6. Stacked histogram presenting potential transport counts (dark grey) and unanimous transport counts (light grey) as a function of wind speed. Dots denote the ratio of the two counts. Dashed line corresponds to minimum wind speed where at least two users observed transport.
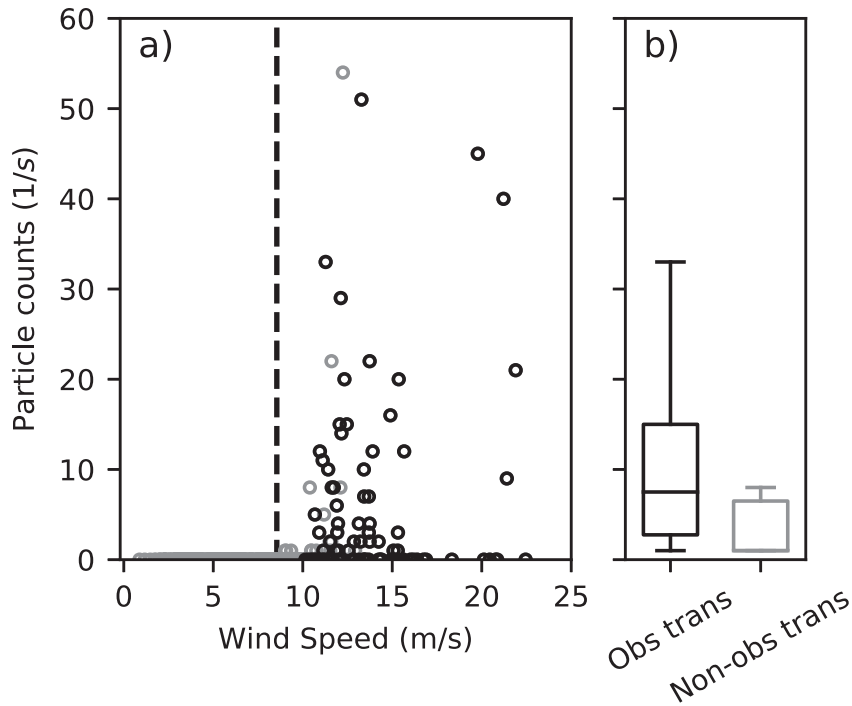

Fig. 7. (a) Scatter plot of $10 \mathrm{~min}$ averaged wind speed against $10 \mathrm{~min}$ averaged particle counts associated with image frames. Black/grey circles correspond to counts in which the majority of users observed/did not observe transport. (b) Corresponding box plot of non-zero particle counts.

transport. The distribution of the nonzero particle counts are presented in Fig. 7b. The non-observable transport counts are positively skewed, with both the 25th percentile and median equal 1 count $\mathrm{s}^{-1}$. The 75 th percentile has a value of 6.5 counts $\mathrm{s}^{-1}$. In contrast, the spread of the observable transport particle counts is larger and occurs at increased particle counts, with the values of the 25th percentile, median and 75th percentile equal to $2.75,7.5$ and 15 counts $\mathrm{s}^{-1}$ respectively.

Fig. 8 indicates that elevated particle counts typically occur when wind speeds exceed $10 \mathrm{~m} / \mathrm{s}$ over an extended period of time, giving rise to a number of discernible transport events. Transport events are typically observed in images throughout their duration. This is highlighted by Table 2 which summarises the counts of images with observable transport per day during the field campaign. Two outliers are observed in the nonzero particle counts associated with non-observable transport. The largest occurred on October 7, 14:00 UTC. At 54 counts $\mathrm{s}^{-1}$, the count is the largest associated with any image across the entire field campaign. However, it also represents the day's only nonzero particle count in which transport was not observed. The second outlier occurs on September 21 at 06:30 UTC ( 22 counts $\mathrm{s}^{-1}$ ). On this day, 5 images taken between 06:00 and 10:30 were associated with non-zero particle counts, three of which overlap with the upper $50 \%$ of nonzero particle counts associated with observable transport.

\subsection{Method performance}

\subsubsection{Validation}

Fig. 9 presents a series of images ((a) August 10 18:00 UTC (b) October 21 13:00 UTC (c) August 30 12:30 UTC) illustrating the grid points about which plan view images were extracted and linear image were features identified. The users unanimously observed transport in each image. The lines passing through the grid points illustrate the orientation of respective image features. The lines are first defined across the respective plan view image, and then projected into the original image. Consequently, they have real world lengths of $50 \mathrm{~m}$. The arrow heads point in the determined propagation direction. The locations and orientations of the linear image features determined by method correspond well with visual observations of streamers.

Given the real world sizing of the plan view images, the real world orientations of the identified linear image features within a given image are expected to cluster around the measured wind direction. To examine this, Fig. $9 \mathrm{~d}$ presents the distribution of the orientations found in 

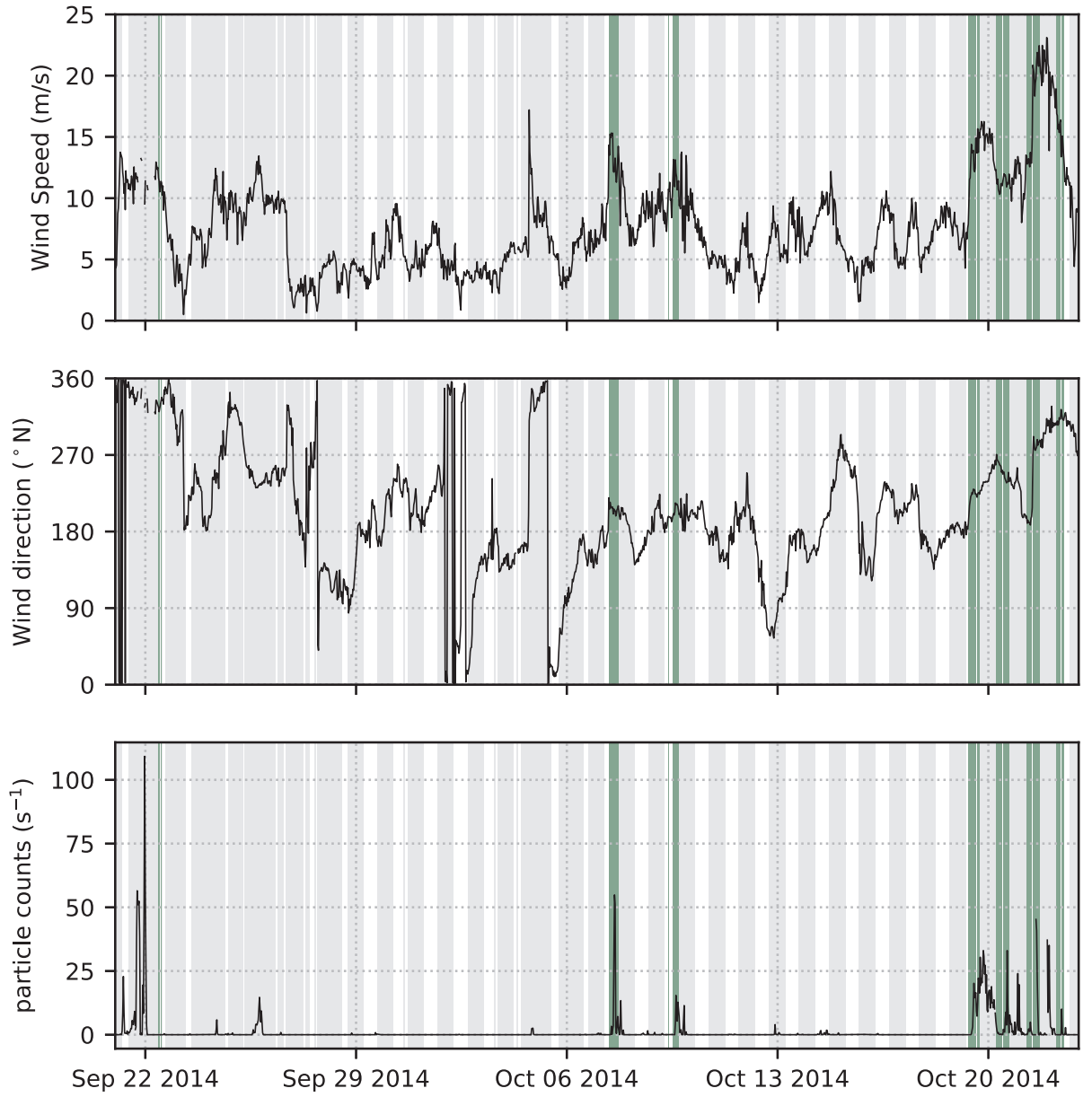

Fig. 8. Time series of $10 \mathrm{~min}$ averaged (a) wind speed (b) wind direction and (c) particle counts plotted on the hour and half hour for visual clarity. Grey bars denote periods where no images are available (i.e. night time hours or operational failure of the camera system). The camera system suffered from a number of technical problems between September 22 and September 29 and consequently returned relatively few images during this period. Green bars indicate periods where the majority of users observed transport in images.
Table 2

Per day counts of images with observed transport.

\begin{tabular}{ll}
\hline Day $(\mathrm{mm} / \mathrm{dd})$ & Counts \\
\hline $09 / 22$ & 3 \\
$10 / 07$ & 13 \\
$10 / 09$ & 11 \\
$10 / 19$ & 16 \\
$10 / 20$ & 16 \\
$10 / 21$ & 18 \\
$10 / 22$ & 11 \\
\hline
\end{tabular}

Fig. 9 a, b, and c. Across each image, the orientations are distributed across a relatively narrow range angles, with the largest range being $10.80^{\circ}$ (August 10). For each image, the average orientation of the identified linear image features is consistent with, albeit slightly in excess of, the measured wind direction. Fig. 10 presents a comparison of the image averaged orientations and the measured wind direction across all images which are classified as containing transport. Consistent with Fig. 9, the image averaged orientations for images which are as containing transport by both the method and the majority of users are typically slightly in excess of the measured wind direction, with the average offset $5.34^{\circ}$. The apparent absence of transport between $0^{\circ} \mathrm{N}$ and $180^{\circ} \mathrm{N}$ is primarily a consequence of the local wind climate, with relatively few winds with speeds in excess of the majority user threshold coming from this direction (see Fig. 11).

\subsection{Performance relative to user results}

The developed method is a binary classifier which predicts an image to be true (i.e. transport observed) or false (i.e. transport not observed). If the predicted class is in agreement with the majority user decision, there are two possible outcomes; either both are true (a true positive) or false (a true negative). Similarly, there are two outcomes if the method is in disagreement with the majority user decision depending on whether the prediction is true (a false positive) or false (a false negative). Fig. 12 presents a confusion matrix which provides an overview of the total counts of the above outcomes across the entire image dataset. A number of measures can be derived from the confusion matrix which allow for the quantification of the methods performance. The measures considered here and their formulations are given in Table 3.

Accuracy describes the probability that the method is in agreement with the majority user decision and is used to evaluate the methods its performance across all examined images. With an accuracy of 0.96 the method is almost always in agreement with the majority user decision. This primarily shows that it performs well in classifying images in which transport is not observed. Therefore, the steps taken to remove plan view images that contain features linear features unrelated to streamers are largely successful.

To examine the methods competence in classifying images with observable transport, precision, recall and the $F 1$ score are considered. Precision describes the probability of an image classified as containing transport by the method also being classified as containing transport by the majority of users and so evaluates the quality of the method's transport classifications. In contrast, recall describes the probability of an image being classified as containing transport by the majority of users also being classified as containing transport by the method, and is used to assess the methods ability to classify transport. A high value of recall doesn't necessarily correspond with a high value for precision. For example, if the method correctly classifies only one image with 

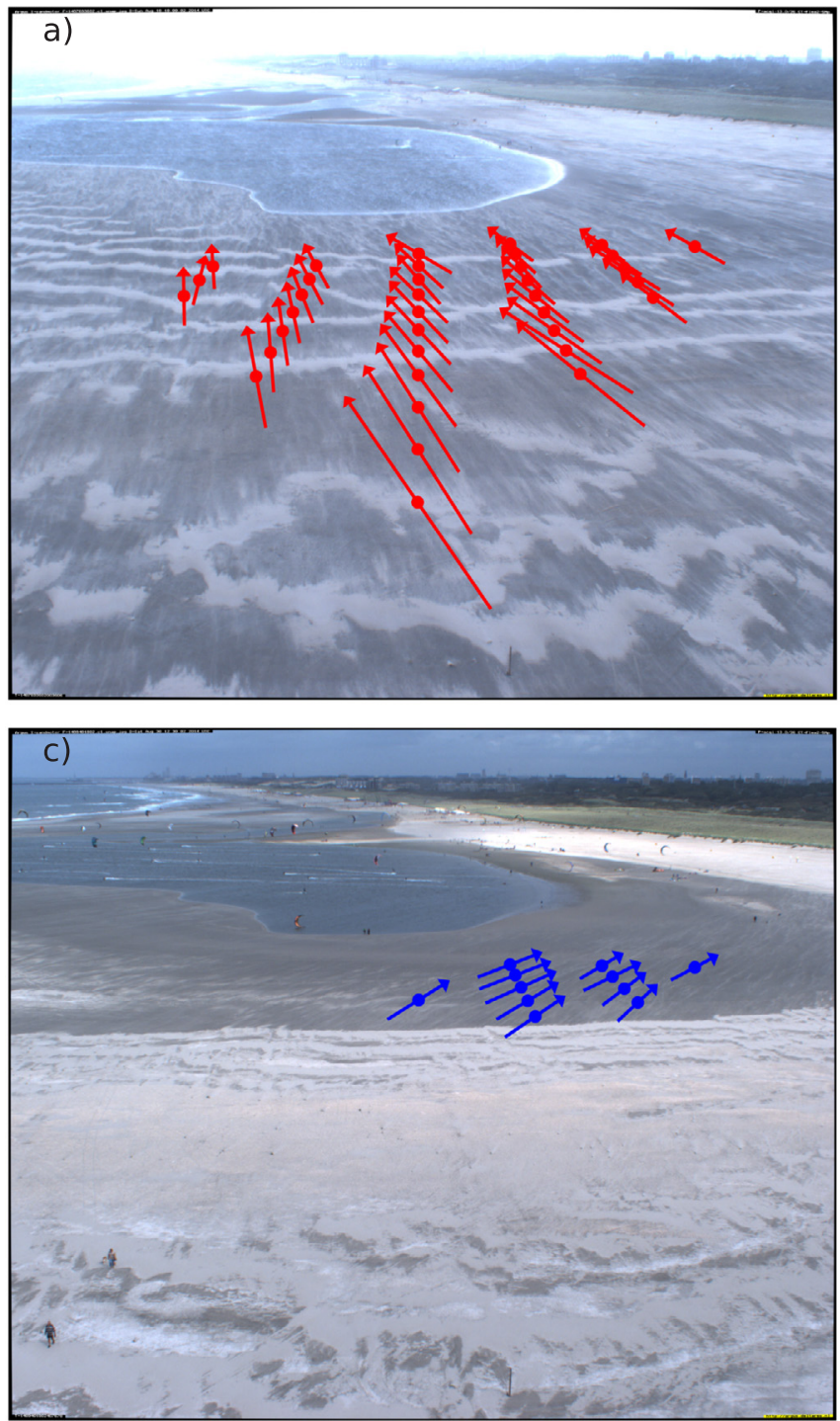

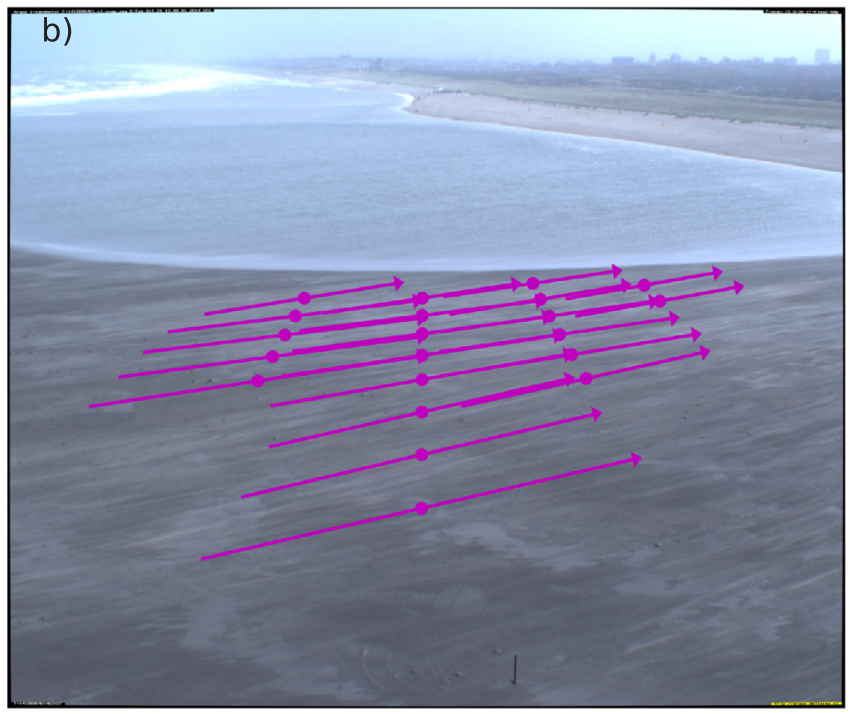

d)

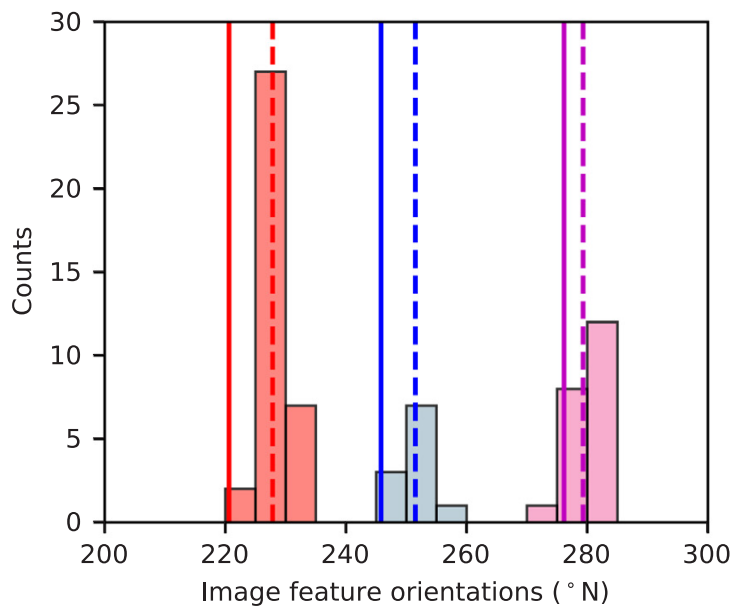

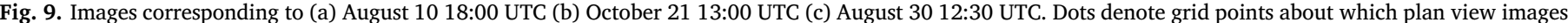

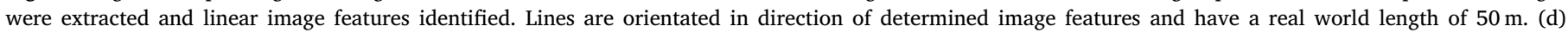

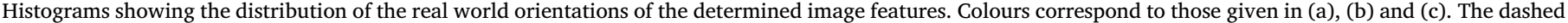
lines highlight the image averaged direction. Solid lines denote the mean wind direction.

transport it will have a precision of 1 but a recall of $\approx 0$. To this end, the $F 1$ score is considered which is the harmonic mean of precision and recall values and so describes the balance of the method. The method performs relatively well in identifying images containing observable transport, with the values of precision, recall, and $F 1$ score all $\geqslant 0.85$. For comparison, Table 3 provides the same measures for each user relative to the majority user decision. It should be stressed that the majority decision is dependent on the individual user results. The results of the developed method are comparable to the lower end of the individual user results.

\section{Discussion}

Previous efforts to identify wind-driven transport events in coastal images have primarily focused on identifying the movement of bedforms between consecutive image frames (e.g. Hage et al., 2018). However, their exact relation to measured transport remains unclear. In this regard, examining images for streamers which can be reconciled with increases in measured particle counts is preferable. The increases in particle counts associated with images containing streamers are likely to be underestimated as a consequence of saturation of the
Wenglor sensors (Hugenholtz and Barchyn, 2011; Sherman et al., 2011; Duarte-Campos et al., 2017). Given the unanimity in the user results, streamers offer a readily identifiable visual signal of transport. In order to observe streamers in images, it is crucial that there is sufficient contrast difference with the underlying beach. Groundwater processes combined with regular inundation will ensure these conditions are met across many beaches. A number of studies present images of streamers in non-rectified images taken around ground level (e.g. see Fig.1 of Baas and Sherman, 2005 or Fig. 4 of Sherman et al., 2013) suggesting that routine RGB coastal images are suitable for resolving transport events at other sites. Consistent with the findings of Delgado-Fernandez (2011), the particle counts indicate that at monthly timescales, wind-driven sediment supply is dominated by a few large-scale transport events which are documented in coastal images. The results presented in this study suggest that routine coastal imagery can be used to resolve transport events for however long the camera is operational.

The method presented in Section 3.2.1 and 3.2.2 for the extraction of plan view images and their subsequent assessment for linear image features is generic if (1) the camera pose is solved (2) the $z$ values of the bounding box are known and (3) there wind data is available. The pose of a camera is readily solved if there are a number ground control points 


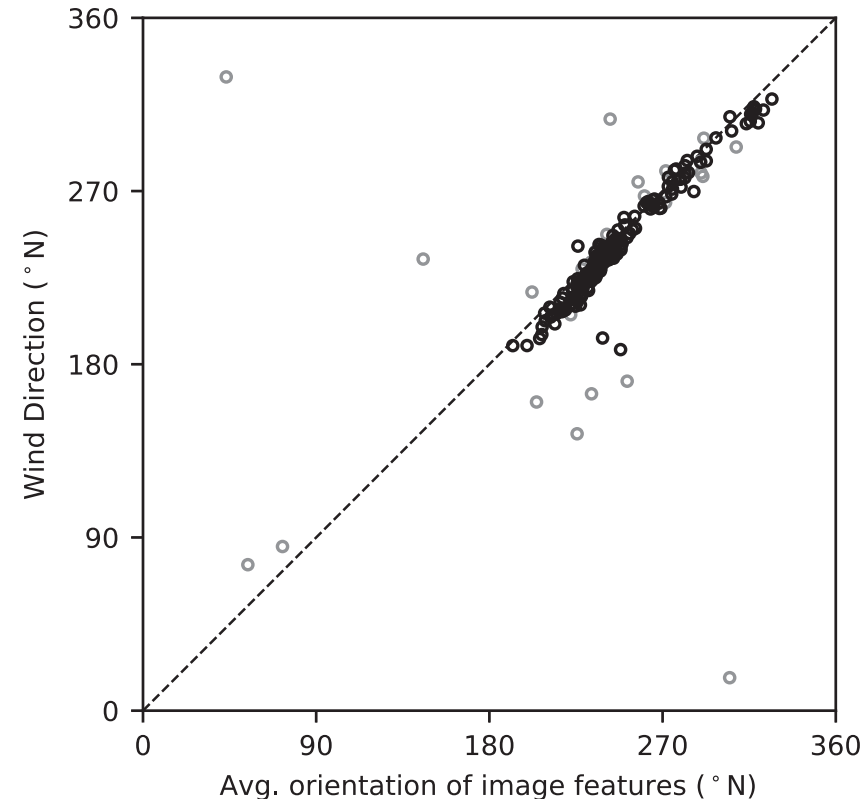

Fig. 10. Comparison of the image averaged orientation of identified linear image features and 10 min averaged wind direction and mean wind direction across all frames in which the method has identified transport. Black dots correspond to true positives, and grey dots false positives. Definition of true/ false positive made relative to the majority user decision. Dashed line illustrates a 1:1 relationship.

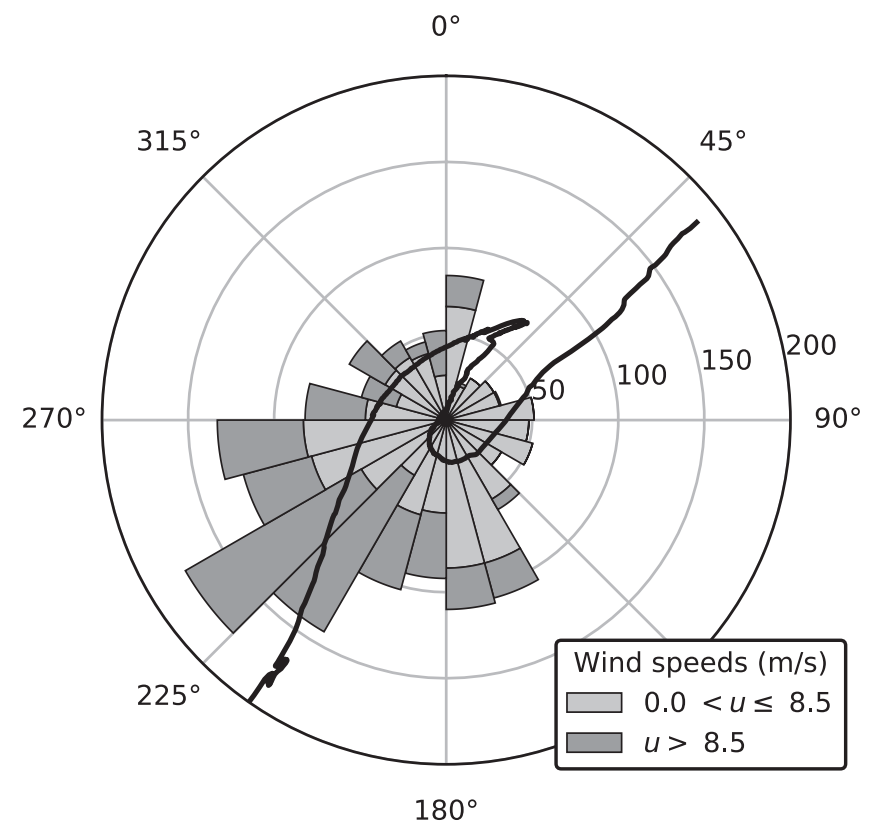

Fig. 11. Wind rose plot of $10 \mathrm{~min}$ averaged wind directions and speeds associated with all image frames considered in this study. Division of wind speeds based on the majority decision for the threshold velocity. The black line represents the outline of the Sand Motor, highlighting its orientation relative to North.

(GCPs) within the view of the camera whose image and real world coordinates are known and whilst it is preferable to get the $z$ values of bounding box corners from a recent topographic survey, they may also be set to a realistic value (Hage et al., 2018). Consequently, the use of wind data is probably the main limiting factor in the general applicability of the presented method. However, it seems likely that if a camera setup is being tailor made for aeolian studies, wind data will also be collected (Delgado-Fernandez, 2011). If a binary response for

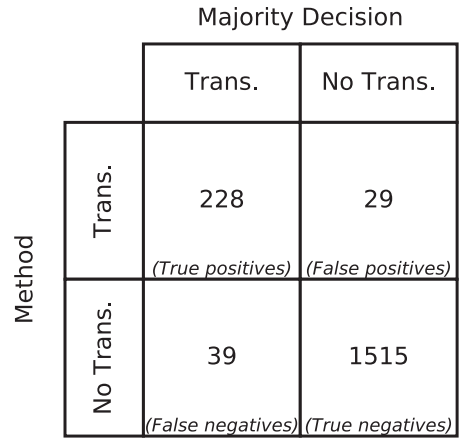

Fig. 12. Confusion matrix comparing the method results to the majority user decision.

Table 3

Summary of the developed methods performance relative to the majority user decision. $t_{p}$ and $f_{p}$ denotes true and false positives respectively.

\begin{tabular}{llllll}
\hline & Definition & \multicolumn{4}{c}{ Value } \\
\cline { 3 - 6 } & & Method & User 1 & User 2 & User 3 \\
\hline Accuracy & $\frac{t_{p}+t_{n}}{t_{p}+f_{p}+f_{n}+t_{n}}$ & 0.96 & 0.99 & 0.99 & 0.99 \\
Precision (P) & $\frac{t_{p}}{t_{p}+f p}$ & 0.88 & 1.00 & 0.98 & 0.91 \\
Recall (R) & $\frac{t_{p}}{t_{p}+f n}$ & 0.85 & 0.91 & 0.97 & 1.0 \\
F1 score & $\frac{2 \mathrm{P} \cdot \mathrm{R}}{P+R}$ & 0.87 & 0.95 & 0.97 & 0.95 \\
\hline
\end{tabular}

transport is required and it is assumed that streamers are the primary source of linear image features, the regression approach can be replaced by a principal component regression which would mitigate the need for wind direction altogether. Within this study, consideration of the wind direction was required to highlight that this assumption is fulfilled.

It remains unclear why the image determined orientations associated with streamers are typically excess of the mean wind direction. Topographic steering may be a contributing factor. For example, field measurements indicate that near-surface airflow can differ significantly from regional airflow, particularly in the vicinity of the dunes (e.g. Walker et al., 2009; Delgado-Fernandez et al., 2013). On more seaward portions of beaches, airflow tends to be directed towards the dune crest (Bauer et al., 2012; Lynch et al., 2009) suggesting that the streamer orientations should be less than the wind direction for winds approaching between $225^{\circ} \mathrm{N}$ and $315^{\circ} \mathrm{N}$ which contrasts with current findings. Potentially, the unusual form and topography of the Sand Motor may contribute to this. Alternatively, the offset may have a more mundane origin, e.g. due to the misalignment of the wind vane or the use of an outdated camera pose. Thermal and wind effects result in the camera pose changing through time and should, therefore, be solved regularly (Holman and Stanley, 2007). Unfortunately, the absence of fixed GCPs within the view of the camera at the site does not permit this. Temporary GCPs are only occasionally deployed across the surface beach and, consequently, the camera pose considered in this study is at best 220 days outdated.

Fig. 9, 10 and 12 highlight that the identification of linear image features is almost always associated with streamers. However, given the complexity of natural scenes, the detection of some linear image features unrelated to streamers is unavoidable. To explore this further, all false positive images were re-examined for suspected causes by User 1 . These are detailed in Table 4. Excluding streamers which were missed by the majority of users, the largest source of false positives are tyre tracks. Contrary to initial expectations, snap images do not correspond to the final image frame of the timex averaging period. Consequently, tyre tracks are occasionally only observable in the timex images, forcing directionality in the spectra through which they pass. When observed in 
Table 4

Suspected causes of false positives.

\begin{tabular}{ll}
\hline Cause & Counts \\
\hline Streamers & 6 \\
Tyre tracks & 6 \\
Standing Water & 5 \\
Bedforms & 5 \\
People & 3 \\
Other & 4 \\
\hline
\end{tabular}

both the snap and timex images, tyre tracks are typically filtered out of the colour difference image. Therefore, these false identifications could be mitigated in the future if it is ensured that the snap image corresponds to the final image frame of the timex averaging periods. After tyre tracks, the joint largest causes of false positives are patches of standing water and bedforms. Standing water typically forms after periods of very high tide and can result in image glare which gets warped across the plan view image. Consequently, variability in the degree of glare between the snap and timex images can introduce directionality into the colour difference images. Brightness differences between snap and timex images across relatively linear bedforms (e.g. see Fig. 9a) composed of dry sediment can also induce directionality across portions of the image where they observed.

To the best of authors knowledge, this paper represents the first time that an automated approach has been adopted to identify aeolian transport in coastal images. The results highlight that exploiting the linear appearance of streamers within images can be a powerful method of identifying images containing transport. With regards to the presented method, its precision, recall, and $F 1$ score indicate that there is room for improvement. Undoubtedly, the classification of plan view images containing streamers would benefit from the inclusion of additional, relevant, image features. For example, additional textural measures can be obtained from the gray level co-occurence matrices and Gabor filters, both of which can be optimised according to the direction determined by the current implementation of the method. Further, including measures related to the intrinsic colour of the plan view image may e.g. limit the identification of linear features within standing patches of water which have very different colours to the underlying beach. The inclusion of an increasing number of features makes manually determining thresholds more challenging and may therefore be better suited to a machine learning framework (e.g. Hoonhout et al., 2015).

\section{Conclusions}

Our ability to predict longer term aeolian sediment supply in coastal settings is hindered by a lack of understanding as to when the transport system is shutdown. Therefore, long-term datasets which are able to document the occurrence of large scale transport events are desirable. This paper illustrates that coastal imagery provides a viable method of achieving this. Streamers provide a readily identifiable visual signal within coastal images that allows transport to be identified. Images containing observable transport are typically associated with elevated particle counts and are observed throughout the duration of significant transport events. Manually classifying images for transport is a timeconsuming activity. To this end, an automated approach is presented in this paper. Given the linear appearance of streamers in images, the method extracts a series of plan-view images from an image frame and examines them for linear image features using 2D-DFTs. The results indicate that textural image information can be utilised successfully to identify images containing transport. Relative to the manually classified dataset, the automated method performs well in identifying images containing transport, with values of precision, recall and $F 1 \geqslant 0.85$. The classification of the plan-view images would likely be improved in the future by the consideration of additional, relevant image features.

\section{Acknowledgements}

The authors thank Bas Hoonhout for making the field data and related scripts available, and for being forthcoming to all related questions. The efforts of Daan Poppema in manually examining the image dataset are greatly appreciated. The authors thank the anonymous reviewer for their extensive feedback which significantly improved the original manuscript. The image and LiDAR datasets are owned by Rijkwaterstaat. This work contributes to the NatureCoast program which is supported by the Dutch Technology Foundation STW: Grant 12686.

\section{Appendix A. Supplementary data}

Supplementary data associated with this article can be found, in the online version, athttps://doi.org/10.1016/j.aeolia.2018.09.003.

\section{References}

Aarninkhof, S.G., Turner, I.L., Dronkers, T.D., Caljouw, M., Nipius, L., 2003. A videobased technique for mapping intertidal beach bathymetry. Coastal Eng. 49 (4), 275-289.

Baas, A.C.W., Sherman, D.J., 2005. Formation and behavior of aeolian streamers. J. Geophys. Res. 110 (F3)

Bagnold, R.A., 1941. The physics of blown sand and dunes. Methuten.

Bauer, B.O., Davidson-Arnott, R.G.D., Walker, I.J., Hesp, P.A., Ollerhead, J., 2012. Wind direction and complex sediment transport response across a beach-dune system. Earth Surface Processes Landforms 37 (15), 1661-1677.

Bretagnon, P., Francou, G., 1988. Planetary theories in rectangular and spherical variables - vsop 87 solutions. Astronomy Astrophys. 202 (1-2), 309-315.

Darke, I., Davidson-Arnott, R., Ollerhead, J., 2009. Measurement of beach surface moisture using surface brightness. J. Coastal Res. 248-256.

Davidson-Arnott, R., Bauer, B., 2009. Aeolian sediment transport on a beach: thresholds, intermittency, and high frequency variability. Geomorphology 105 (1), 117-126.

Davidson-Arnott, R.G.D., Law, M.N., 1996. Measurement and prediction of long-term sediment supply to coastal foredunes. J. Coastal Res. 12 (3), 654-663.

Delgado-Fernandez, I., 2010. A review of the application of the fetch effect to modelling sand supply to coastal foredunes. Aeolian Res. 2 (2), 61-70.

Delgado-Fernandez, I., 2011. Meso-scale modelling of aeolian sediment input to coastal dunes. Geomorphology 130 (3), 230-243.

Delgado-Fernandez, I., Davidson-Arnott, R., 2010. Meso-scale aeolian sediment input to coastal dunes: the nature of aeolian transport events. Geomorphology 126 (1), 217-232.

Delgado-Fernandez, I., Davidson-Arnott, R., Ollerhead, J., 2009. Application of a remote sensing technique to the study of coastal dunes. J. Coastal Res. 1160-1167.

Delgado-Fernandez, I., Jackson, D.W.T., Cooper, J.A.G., Baas, A.C.W., Beyers, J.H.M., Lynch, K., 2013. Field characterization of three dimensional lee side airflow patterns under offshore winds at a beach dune system. J. Geophys. Res.: Earth Surface 118 (2), 706-721.

Duarte-Campos, L., Wijnberg, K.M., Oyarte-Gálvez, L., Hulscher, S.J.M.H., 2017. Laser particle counter validation for aeolian sand transport measurements using a highspeed camera. Aeolian Res. 25, 37-44.

Ellis, J.T., Sherman, D.J., Farrell, E.J., Li, B., 2012. Temporal and spatial variability of aeolian sand transport: Implications for field measurements. Aeolian Research 3 (4), 379-387 [The 7th International Conference on Aeolian Research (ICAR VII), Santa Rosa, Argentina].

Hage, P., Ruessink, B., Donker, J., 2018. Determining sand strip characteristics using argus video monitoring. Aeolian Res. 33, 1-11.

Hartley, R.I., Zisserman, A., 2004. Multiple View Geometry in Computer Vision, 2nd edition. Cambridge University Press.

Holman, R., Stanley, J., 2007. The history and technical capabilities of Argus. Coastal Eng. 54, 477-491.

Hoonhout, B., de Vries, S., 2017a. Aeolian sediment supply at a mega nourishment. Coastal Eng. 123 (Supplement C), 11-20.

Hoonhout, B., de Vries, S., 2017b. Field measurements on spatial variations in aeolian sediment availability at the sand motor mega nourishment. Aeolian Res. 24, 93-104.

Hoonhout, B., Radermacher, M., Baart, F., van der Maaten, L., 2015. An automated method for semantic classification of regions in coastal images. Coastal Eng. 105, $1-12$.

Hsu, S.-A., 1974. Computing eolian sand transport from routine weather data. In: Coastal Engineering Proceedings. No. 14. ACSE, pp. 1619-1626.

Hugenholtz, C.H., Barchyn, T.E., 2011. Laboratory and field performance of a laser particle counter for measuring aeolian sand transport. J. Geophys. Res.: Earth Surf. 116 (F1).

Josso, B., Burton, D.R., Lalor, M.J., 2005. Texture orientation and anisotropy calculation by fourier transform and principal component analysis. Mech. Syst. Signal Process. 19 (5), 1152-1161.

Kawamura, R., 1951. Study on sand movement by wind. Tech. rep., Reports of Physical Sciences Research Institute of Tokoyo University.

Keijsers, J.G.S., Poortinga, A., Riksen, M.J.P.M., Maroulis, J., 2014. Spatio-temporal 
variability in accretion and erosion of coastal foredunes in the netherlands: regional climate and local topography. PLOS ONE 9 (3), 1-11.

Lynch, K., Jackson, D.W., Cooper, J.A.G., 2009. Foredune accretion under offshore winds. Geomorphology 105 (1), 139-146.

Sherman, D.J., Houser, C., Ellis, J.T., Farrell, E.J., Li, B., Davidson-Arnott, R.G., Baas, A.C., Maia, L.P., 2013. Characterization of aeolian streamers using time-average videography. J. Coastal Res., SI 65, 1331-1336.

Sherman, D.J., Li, B., 2012. Predicting aeolian sand transport rates: a reevaluation of models. Aeolian Res. 3 (4), 371-378.

Sherman, D.J., Li, B., Farrell, E.J., Ellis, J.T., Cox, W.D., Maia, L.P., Sousa, P.H.G.O., 2011. Measuring aeolian saltation: a comparison of sensors. J. Coastal Res. 280-290.

Stive, M., de Schipper, M., Luijendijk, A., Aarninkhof, S., van Gelder-Maas, C., van Thiel de Vries, J., de Vries, S., Henriquez, M., Marx, S., Ranasinghe, R., 2013. A new alternative to saving our beaches from sea-level rise: the Sand Engine. J. Coastal Res. 29 (5), 1001-1008.

van der Wal, D., 1998. Effects of fetch and surface texture on aeolian sand transport on two nourished beaches. J. Arid Environ. 39 (3), 533-547.

van der Weerd, A.J., Wijnberg, K.M., 2016. Aeolian sediment flux derived from a natural sand trap. J. Coastal Res. 75, 338-342.

Walker, I.J., Hesp, P.A., Davidson-Arnott, R.G., Bauer, B.O., Namikas, S.L., Ollerhead, J., 2009. Responses of three-dimensional flow to variations in the angle of incident wind and profile form of dunes: Greenwich Dunes, Prince Edward Island. Canada. Geomorphology 105 (1), 127-138.

Wiggs, G., Baird, A., Atherton, R., 2004. The dynamic effects of moisture on the en trainment and transport of sand by the wind. Geomorphology 59 (1), 13-30. 